\title{
A simple numerical scheme to linear radiative transfer in hollow and solid spheres
}

\section{Um esquema numérico simples para transferência radiativa linear em esferas ocas e maciças}

\author{
Cibele Aparecida Ladeia ${ }^{1}$; Marcelo Schramm² \\ Julio Cesar Lombaldo Fernandes ${ }^{3}$
}

\begin{abstract}
In this work we consider the linear radiative transfer in hollow and solid spheres and the solution in a medium with diffusely reflecting boundaries and energy source as well. The discrete ordinates method with diamond difference scheme is used to calculate the radiation intensities and the partial heat fluxes at the boundaries. Our results were obtained for forward, isotropic and backward scattering and were compared with data in the literature.
\end{abstract}

Keywords: Linear radiative transfer. Hollow and solid spheres. Discrete ordinates method. Diamond difference.

\section{Resumo}

Nesse trabalho consideramos a transferência radiativa linear em esferas ocas e maciças, bem como a solução em um meio com fronteiras refletoras difusas e com fonte de energia. O método de ordenadas discretas com a técnica de diamond difference é utilizado para calcular as intensidades de radiação e os fluxos de calor parciais nas fronteiras. Nossos resultados foram obtidos para os espalhamentos para frente, isotrópico e para trás e foram comparados com os dados da literatura.

Palavras-chave: Transferência radiativa linear. Esferas ocas e maciças. Método de ordenadas discretas. Diamond difference.

\footnotetext{
${ }^{1}$ Prof. Dra., Depto. Matemática Pura e Aplicada, UFRGS, Porto Alegre, RS, Brasil, E-mail: cibele.ladeia@ufrgs.br

2 Prof. Dr., Prog. de Pós-Graduação em Modelagem Matemática, UFPel, Pelotas, RS, Brasil, E-mail: schrammmarcelo@gmail.com

${ }^{3}$ Prof. Dr., Depto. Matemática Pura e Aplicada, UFRGS, Porto Alegre, RS, Brasil, E-mail: julio.lombaldo@ufrgs.br
} 


\section{Introduction}

The radiative transfer equation in spherical geometry has numerous applications. Examples include, among others, the stellar atmosphere, furnaces and nuclear reactors (HOWELL; MENGUC; SIEGEL, 2016; MODEST, 2013). There are different techniques to solve the radiative transfer in spheres, for example Thynell and Ozisik (1985) used the Galerkin technique to simulate the radiative transfer in solid spheres for isotropic scattering; and Tsai et al. (1989) used the finite volumes method and the discrete ordinates method in inhomogeneous and anisotropic scattering solid and hollow spheres. In a first moment, Abulwafa (1993) applied a Galerkin technique to hollow spheres, and later Abulwafa and Attia (1997) used the classical Pomraning-Eddington technique to obtain a semi-analytical solution for solid spheres, both papers for linearly anisotropic scattering. Sghaier (2013) used a moment method (SGHAIER, 2013), which reduces scattering angular difficulties, but only in hollow spheres. Elghazaly (2009) also used the Pomraning-Eddington technique to obtain the solution for solid spheres (ELGHAZALY, 2009), but for different (more accurate) boundary conditions.

As it is noteworthy, most of the researchers in the field of linear radiative transfer in spherical geometry use numerical methods, not always for both solid and hollow spheres, also not always for anisotropic scattering. In this work, we present a simple numerical method to solve the linear radiative transfer in hollow and solid spheres with anisotropic scattering.

We use the discrete ordinates method with a diamond difference scheme (CHANDRASEKHAR, 1950; LEWIS; MILLER JUNIOR, 1984). This combination is quite usual in numerical methodologies for radiation transfer problems. We use the discrete ordinates method in the angular variable and a numerical integration over a grid in the spatial variable. The finite volumes method also requires an numerical integration, however we propose a different integration rule, where the nodes are in the edges of each segment (not in the center). This procedure produces a mesh, over which the radiation intensity assumes constant values. Following the diamond difference scheme, we solve the discretized radiation intensities one by one, in an iterative routine. Finally, we report cases with numerical solutions that are compared with data in the literature.

\section{Radiative transfer equation in spherical geome- try}

We consider the radiative transfer equation in spherical geometry (OZISIK, 1973),

$$
\begin{aligned}
\frac{\mu}{r^{2}} \frac{\partial}{\partial r}\left[r^{2} I\right]+ & \frac{1}{r} \frac{\partial}{\partial \mu}\left[\left(1-\mu^{2}\right) I\right] \\
& +I=(1-\omega(r)) I_{b}(T) \\
& +\frac{\omega(r)}{2} \int_{-1}^{1} P\left(\mu, \mu^{\prime}\right) I\left(r, \mu^{\prime}\right) d \mu^{\prime},
\end{aligned}
$$

where $r \in[a, b], 0 \leq a<b$, is the optical (space) variable and $\mu \in[-1,1]$. Here, $\mu=\cos \theta, \theta$ is the direction polar angle and $\mu^{\prime}$ is the direction cosine of the incident rays. $I=I(r, \mu)$ is the radiation intensity, $I_{b}(T)$ is the black body radiation for temperature $T, \omega$ is the single scattering albedo and $P$ is the phase function, a relation between the incident and scattered radiations. $P$ may be approximated as a truncated series with a Legendre polynomials basis (CHANDRASEKHAR, 1950),

$$
P\left(\mu, \mu^{\prime}\right)=\sum_{\ell=0}^{L} \beta_{\ell} \mathscr{P}_{\ell}(\mu) \mathscr{P}_{\ell}\left(\mu^{\prime}\right),
$$

where $\beta_{\ell}$ are the expansion coefficients of the Legendre polynomials and $\mathscr{P}_{\ell}$ is the $\ell$-th Legendre polynomial. Here, $L$ refers to the degree of anisotropy. Also, if $\beta_{1}>0$ or $\beta_{1}<0$ we say $P$ is a forward or backward scattering phase function, respectively; and if $\beta_{\ell}=0$ for $\ell \geq 1$ we say it is an isotropic scattering phase function (PETTY, 2006).

The boundary conditions of equation (1) are

$$
I(a, \mu)= \begin{cases}\varepsilon_{1} I_{b 1}(T) & \\ -2 \rho_{1} \int_{-1}^{0} I\left(a, \mu^{\prime}\right) \mu^{\prime} d \mu^{\prime}, & a>0 \\ I(0,-\mu), & a=0\end{cases}
$$

for $\mu>0$, and

$$
I(b, \mu)=\varepsilon_{2} I_{b 2}(T)+2 \rho_{2} \int_{0}^{1} I\left(b, \mu^{\prime}\right) \mu^{\prime} d \mu^{\prime}
$$

for $\mu<0$. Here, $\varepsilon_{1}$ and $\varepsilon_{2}$ are the emissivities at the inner and outer radii, respectively. Similarly, $\rho_{1}$ and $\rho_{2}$ are the diffusive reflectivities at the inner and outer radii, respectively; and $I_{b 1}(T)$ and $I_{b 2}(T)$ are the black body radiations for temperature $T$ at the inner and outer radii, respectively. 
In this paper, we use equation (1) in the form

$$
\begin{aligned}
\mu \frac{\partial}{\partial r} I+\frac{2 \mu}{r} I+ & \frac{1}{r} \frac{\partial}{\partial \mu}\left[\left(1-\mu^{2}\right) I\right] \\
+ & I=(1-\omega(r)) I_{b}(T) \\
& +\frac{\omega(r)}{2} \int_{-1}^{1} P\left(\mu, \mu^{\prime}\right) I\left(r, \mu^{\prime}\right) d \mu^{\prime}
\end{aligned}
$$

for $(r, \mu) \in[a, b] \times[-1,1]$.

\section{Discrete ordinates method}

The discrete ordinates method $S_{N}$ (CHANDRASEKHAR, 1950) is a classical method based on the angular variable discretization in an enumerable set of angles, or equivalently, their direction cosines. In our work, $\left\{\mu_{m}\right\}_{1}^{M}$ where $M$ is the amount of discrete ordinates. We use the $S_{N}$ technique in the angular variable, so we define $I_{m}(r)=I\left(r, \mu_{m}\right)$. The $S_{N}$ form of equation (5) is

$$
\begin{aligned}
\mu_{m} \frac{d}{d r} I_{m}+\frac{2 \mu_{m}}{r} I_{m} & +\frac{1}{r} \frac{\alpha_{m+1 / 2} I_{m+1 / 2}-\alpha_{m-1 / 2} I_{m-1 / 2}}{w_{m}} \\
+I_{m} & =(1-\omega(r)) I_{b}(T) \\
& +\frac{\omega(r)}{2} \sum_{m^{\prime}=1}^{M} w_{m^{\prime}} P\left(\mu_{m}, \mu_{m^{\prime}}\right) I_{m^{\prime}}
\end{aligned}
$$

for $m=1,2, \ldots, M$, where $\mu_{m}$ and $w_{m}$ are the (crescent) abscissas and weights of a quadrature rule and $\alpha_{m \pm 1 / 2}$ are obtained by the recursion formula (LEWIS; MILLER JUNIOR, 1984)

$$
\begin{aligned}
& \alpha_{1 / 2}=0 \\
& \alpha_{m+1 / 2}=\alpha_{m-1 / 2}-2 \mu_{m} w_{m}
\end{aligned}
$$

for $m=1,2, \ldots, M$. Here, the $S_{N}$ method requires an even order quadrature, so $M$ must be even. In this paper, without loss of generality, we use an even order Gauss-Legendre quadrature. Also, note that the integral term in (5) is approximated as a sum due to the quadrature rule to evaluate it. This is one of the great advantages of using the discrete ordinates method.

In order to solve (6) for $I_{m}(r)$ with an iterative scheme for $m=1,2, \ldots, M$, we use the mean (LEWIS; MILLER JUNIOR, 1984)

$$
I_{m+1 / 2}=2 I_{m}-I_{m-1 / 2}
$$

and we rewrite the equation (6) as

$$
\begin{gathered}
\mu_{m} \frac{d}{d r} I_{m}+\frac{2 \mu_{m}}{r} I_{m}+\frac{2 \alpha_{m+1 / 2}}{r w_{m}} I_{m}+I_{m} \\
=\frac{\alpha_{m+1 / 2}+\alpha_{m-1 / 2}}{r w_{m}} I_{m-1 / 2}+(1-\omega(r)) I_{b}(T) \\
\quad+\frac{\omega(r)}{2} \sum_{m^{\prime}=1}^{M} w_{m^{\prime}} P\left(\mu_{m}, \mu_{m^{\prime}}\right) I_{m^{\prime}} .
\end{gathered}
$$

To start the iterative scheme, we need $I_{1 / 2}(r)$. To obtain it, we consider equation (5), expand the derivative in $\mu$, cancel some terms out, evaluate it at the discrete ordinate $\mu_{1 / 2}=-1$, use the quadrature rule in the integral over $\mu^{\prime}$ and get

$$
\begin{aligned}
-\frac{d}{d r} I_{1 / 2}+I_{1 / 2} & =(1-\omega(r)) I_{b}(T) \\
& +\frac{\omega(r)}{2} \sum_{m^{\prime}=1}^{M} w_{m^{\prime}} P\left(-1, \mu_{m^{\prime}}\right) I_{m^{\prime}} .
\end{aligned}
$$

The discrete ordinates form of the boundary conditions writes

$$
\begin{aligned}
I_{1 / 2}(b) & =\varepsilon_{2} I_{b 2}(T)+2 \rho_{2} \sum_{m^{\prime}=M / 2+1}^{M} \mu_{m^{\prime}} w_{m^{\prime}} I_{m^{\prime}}(b), \\
I_{m}(b) & =\varepsilon_{2} I_{b 2}(T)+2 \rho_{2} \sum_{m^{\prime}=M / 2+1}^{M} \mu_{m^{\prime}} w_{m^{\prime}} I_{m^{\prime}}(b)
\end{aligned}
$$

for $m=1,2, \ldots, M / 2$, and

$$
I_{m}(a)=\left\{\begin{aligned}
\varepsilon_{1} I_{b 1}(T) & \\
-2 \rho_{1} \sum_{m^{\prime}=1}^{M / 2} \mu_{m^{\prime}} w_{m^{\prime}} I_{m^{\prime}}(a), & a>0 \\
I_{M+1-m}(0), & a=0
\end{aligned}\right.
$$

for $m=M / 2+1, M / 2+2, \ldots, M$.

\section{Spatial discretization}

We discretize the position variable $a \leq r \leq b$ in the nodes $\left\{r_{i}\right\}_{0}^{N}$, wherein $r_{i}=a+i \Delta r$ for $i=0,1, \ldots, N$ and $\Delta r=(b-a) / N=r_{i+1}-r_{i}$ for all $i$ but $N$.

We propose a different approach in this discretization. For example, the finite differences method imply an evaluation of the equation at the nodes, and get an algebraic system where the unknowns are the $I_{m}$ in the edges of the cells; and the finite volumes method requires the integration of the equation over a control volume, and also get an algebraic system where the unknowns are the $I_{m}$ in the center of the cells. In this article we used an integral operator in the equation and got the unknowns in the edges of the cells. 
We apply the operators $\frac{1}{\Delta r} \int_{r_{i}}^{r_{i+1}}(\cdot) r d r$ and $\frac{1}{\Delta r} \int_{r_{i}}^{r_{i+1}}(\cdot) d r$ in equations (10) and (11), respectively, and used the trapezoidal rule, dropping the error term. This results in a grid with nodes in the edges of the segments, obtained by integral operators. Defining $I_{m}^{i}=I_{m}\left(r_{i}\right)$, we have

$$
\begin{aligned}
& {\left[\frac{\mu_{m} r_{i+1}}{\Delta r}+\frac{\mu_{m}}{2}\right] I_{m}^{i+1}+\left[-\frac{\mu_{m} r_{i}}{\Delta r}+\frac{\mu_{m}}{2}\right] I_{m}^{i}} \\
& +\left[\frac{\alpha_{m+1 / 2}}{w_{m}}\right] I_{m}^{i+1}+\left[\frac{\alpha_{m+1 / 2}}{w_{m}}\right] I_{m}^{i}+\left[\frac{r_{i+1}}{2}\right] I_{m}^{i+1} \\
& +\left[\frac{r_{i}}{2}\right] I_{m}^{i}=\left[\frac{\alpha_{m+1 / 2}+\alpha_{m-1 / 2}}{2 w_{m}}\left(I_{m-1 / 2}^{i+1}+I_{m-1 / 2}^{i}\right)\right] \\
& +\left[\frac{r_{i+1}\left(1-\omega\left(r_{i+1}\right)\right) I_{b}(T)+r_{i}\left(1-\omega\left(r_{i}\right)\right) I_{b}(T)}{2}\right] \\
& +\left[\sum_{m^{\prime}=1}^{M} \frac{w_{m^{\prime}} P\left(\mu_{m}, \mu_{m^{\prime}}\right)}{4}\right. \\
& \left.\times\left(r_{i+1} \omega\left(r_{i+1}\right) I_{m^{\prime}}^{i+1}+r_{i} \omega\left(r_{i}\right) I_{m^{\prime}}^{i}\right)\right]
\end{aligned}
$$

and

$$
\begin{aligned}
& {\left[\frac{1}{\Delta r}\right] I_{1 / 2}^{i}+\left[\frac{1}{2}\right] I_{1 / 2}^{i}=\left[\frac{1}{\Delta r}\right] I_{1 / 2}^{i+1}+\left[-\frac{1}{2}\right] I_{1 / 2}^{i+1}} \\
& +\left[\sum_{m^{\prime}=1}^{M} \frac{w_{m^{\prime}} P\left(-1, \mu_{m^{\prime}}\right)}{4}\left(\omega\left(r_{i+1}\right) I_{m^{\prime}}^{i+1}+\omega\left(r_{i}\right) I_{m^{\prime}}^{i}\right)\right] \\
& +\left[\frac{\left(1-\omega\left(r_{i+1}\right)\right) I_{b}(T)+\left(1-\omega\left(r_{i}\right)\right) I_{b}(T)}{2}\right],
\end{aligned}
$$

both for $i=0,1, \ldots, N-1$. For illustration, Figure 1 shows the double discrete variables $(i, m)$ represent the continuous variables $(r, \mu)$ in a mesh.

The boundary conditions (12), (13) and (14) are expressed as

$$
\begin{gathered}
I_{1 / 2}^{N}=\varepsilon_{2} I_{b 2}(T)+2 \rho_{2} \sum_{m^{\prime}=M / 2+1}^{M} w_{m^{\prime}} \mu_{m^{\prime}} I_{m^{\prime}}^{N}, \\
I_{m}^{N}=\varepsilon_{2} I_{b 2}(T)+2 \rho_{2} \sum_{m^{\prime}=M / 2+1}^{M} w_{m^{\prime}} \mu_{m^{\prime}} I_{m^{\prime}}^{N}
\end{gathered}
$$

for $m=1,2, \ldots, M / 2$, and
Figure 1 - A schematic (out of scale) mesh of the $(r, \mu)$ discretization into the variables $(i, m)$ with corresponding values $r \rightarrow i$ and $\mu \rightarrow m$. $I_{m}^{i}$ are positioned above the $(i, m)$ nodes. Also, the ray propagation directions are indicated in the right for some values of $\mu$.

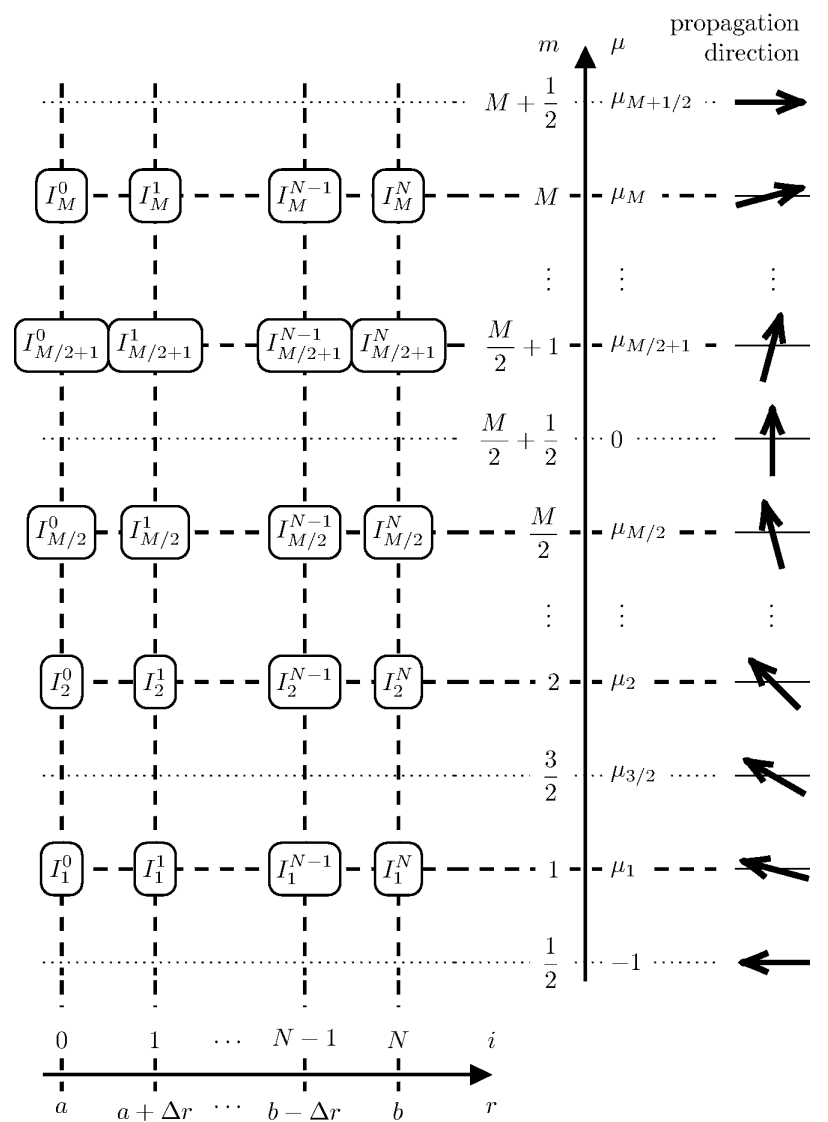

Source: The authors.

$$
I_{m}^{0}=\left\{\begin{aligned}
\varepsilon_{1} I_{b 1}(T) & \\
-2 \rho_{1} \sum_{m^{\prime}=1}^{M / 2} \mu_{m^{\prime}} w_{m^{\prime}} I_{m^{\prime}}^{0}, & a>0 \\
I_{M+1-m}^{0}, & a=0
\end{aligned}\right.
$$

for $m=M / 2+1, M / 2+2, \ldots, M$, respectively.

To obtain the solution of this linear algebraic system, we used a diamond difference scheme, exemplified in Algorithm 1. There, all right-hand sides of the cited equations are computed using older values. Also, we iterate in a diamond difference scheme (LEWIS; MILLER JUNIOR, 1984), id est for $m \leq M / 2$ we update $I_{m}^{i}$ for $i$ going from $N$ to 0 , and for $m>M / 2$ we update $I_{m}^{i}$ for $i$ going from 0 to $N$. That means we are updating the boundaries $I_{m}$ first, and then the rest of the $I_{m}^{i}$ for the same direction angle. 
Algorithm 1: Pseudocode for the present methodology.

Data: Problem data: $a, b, \omega, P, I_{b}(T), \varepsilon_{1}, \rho_{1}$, $I_{b 1}(T), \varepsilon_{2}, \rho_{2}, I_{b 2}(T)$

Data: Numerical data: $M, N$, stop criteria " $\mathrm{O}$ " for the outer iterations and "I" for the inner iterations.

Compute $\mu_{m}, w_{m}, \alpha_{m \pm 1 / 2}, \Delta r, r_{i}$ for all $m=1,2, \ldots, M$ and $i=0,1, \ldots, N$

Make initial guess $I_{m}^{i}$ for all $m=1,2, \ldots, M$ and $i=0,1, \ldots, N$

while stop criterion $\mathrm{O}$ is not satisfied do

Compute $I_{1 / 2}^{N}$ using (17)

for $i=N-1, N-2, \ldots, 0$ do

Solve (16) for $I_{1 / 2}^{i}$

end

for $m=1,2, \ldots, M / 2$ do

while stop criterion I is not satisfied do

Compute $I_{m}^{N}$ using (18)

for $i=N-1, N-2, \ldots, 0$ do

Solve (15) for $I_{m}^{i}$

end

end

for $i=0,1, \ldots, N$ do

Compute $I_{m+1 / 2}^{i}$ using (9)

end

end

for $m=M / 2+1, M / 2+2, \ldots, M$ do

while stop criterion I is not satisfied do

Compute $I_{m}^{0}$ using (19)

for $i=0,1, \ldots, N-1$ do

Solve (15) for $I_{m}^{i+1}$

end

end

for $i=0,1, \ldots, N$ do

Compute $I_{m+1 / 2}^{i}$ using (9)

end

end

end

Result: Matrix with values of $I_{m}^{i}$

\section{Numerical results}

We used the present methodology to solve the linear radiative transfer problem in hollow and solid spheres. For the purpose of comparison with available data in literature (ABULWAFA, 1993; ABULWAFA; ATTIA, 1997), we define backward and forward radiation fluxes as

$$
\begin{aligned}
& q^{-}\left(r_{i}\right)=\sum_{m^{\prime}=1}^{M / 2} w_{m^{\prime}} \mu_{m^{\prime}} I_{m^{\prime}}^{i}, \\
& q^{+}\left(r_{i}\right)=\sum_{m^{\prime}=M / 2+1}^{M} w_{m^{\prime}} \mu_{m^{\prime}} I_{m^{\prime}}^{i} .
\end{aligned}
$$

We used the stop criterion of relative distance to the average between two consecutive iterations,

$$
\max _{\substack{m=1, \ldots, M \\ i=0, \ldots, N}}\left|\frac{I_{m}^{i}-\left(I_{m}^{i}\right)_{\text {old }}}{I_{m}^{i}+\left(I_{m}^{i}\right)_{\text {old }}}\right| \leq 10^{-6},
$$

in both inner and outer loops. Here, the $\left(I_{m}^{i}\right)_{\text {old }}$ represents values of $I_{m}^{i}$ of the previous iteration.

The coefficients $\beta_{\ell}$ in equation (2) are evaluated according to Table 1 (ABULWAFA, 1993). Note that in the reference, the authors used an approximation to linear phase function in their methods. We do not use any sort of approximation in the phase function per se. The approximation in the angular variable occur due to the application of the discrete ordinates method.

Table 1 - Values of $\beta_{\ell}$ in (2) for the phase function of different scattering cases.

\begin{tabular}{lrrr}
\hline$\ell$ & Forward & Isotropic & Backward \\
\hline 0 & 1.00000 & 1.00000 & 1.00000 \\
1 & 1.98398 & 0.00000 & -0.56524 \\
2 & 1.50823 & 0.00000 & 0.29783 \\
3 & 0.70075 & 0.00000 & 0.08571 \\
4 & 0.23489 & 0.00000 & 0.01003 \\
5 & 0.05133 & 0.00000 & 0.00063 \\
6 & 0.00760 & 0.00000 & 0.00000 \\
7 & 0.00048 & 0.00000 & 0.00000 \\
8 & 0.00000 & 0.00000 & 0.00000 \\
\hline
\end{tabular}

Source: Table 1 of Abulwafa (1993) with our zeros and Isotropic column completion.

In the following, we present the numerical results for three cases, Case 1 for hollow sphere, Case 2 for solid sphere and Case 3 for mesh refinement. In all cases, according to the discrete ordinates method, we chose an even integer $M$.

\section{Case 1 - Hollow Sphere}

For this case, we consider homogeneous and inhomogeneous hollow spheres, with numerical values $a=$ 1 and $b=2, P$ is given by equation (2), where the values of $\beta_{\ell}$ are given by Table $1 . \omega$ is given by Table 2 (referenced by $j$ ), $\varepsilon_{1}=\varepsilon_{2}=0.75, \rho_{1}=\rho_{2}=0.25$, 
$I_{b 1}(T)=0, I_{b 2}(T)=4 / 3$ and several $I_{b}(T)$. We consider a mesh with $M=30$ and $N=100$. Tables 3, 4 and 5 show the obtained numerical values of $q^{+}(b)$ and $-q^{-}(a)$ (to show positive values) for forward, isotropic and backward scattering compared with the ones in the Table 6 of Abulwafa (1993). In this case, we use the numerical quantities (ABULWAFA, 1993),

$$
\begin{aligned}
& F=\left(b^{4}-a^{4}\right) /\left(b^{3}-a^{3}\right), \\
& H=\left(b^{5}-a^{5}\right) /\left(b^{3}-a^{3}\right),
\end{aligned}
$$

in the data tables. In the result tables, REF and P.M. refer to "Abulwafa (1993)" and "Present Methodology", respectively.

Table 2 - Different single scattering albedos for case 1

\begin{tabular}{|c|c|c|c|}
\hline $\bar{j}$ & $\omega(r)$ & $j$ & $\omega(r)$ \\
\hline 1 & $2 r / 3 F$ & 7 & $1.0-2 r / 3 F$ \\
\hline 2 & $0.2+2 r / 5 F$ & 8 & $4 r / 15 F+r^{2} / 2 H$ \\
\hline 3 & $0.4+2 r / 15 F$ & 9 & $0.4-4 r / 15 F+r^{2} / 2 H$ \\
\hline 4 & 0.5 & 10 & $0.6-8 r / 15 F+r^{2} / 2 H$ \\
\hline 5 & $0.6-2 r / 15 F$ & 11 & $1.0-16 r / 15 F+r^{2} / 2 H$ \\
\hline 6 & $0.8-2 r / 5 F$ & & \\
\hline
\end{tabular}
( $F$ and $H$ are computed as in equations (23) and (24)).

Source: Table 6 of Abulwafa (1993).

\section{Case 2 - Solid sphere}

For this case, we consider homogeneous solid spheres, with numerical values $a=0$ and several sphere radius $b$. by equation (2), where the values of $\beta_{\ell}$ are given by Table 1 and $\omega$ assumes constant values, from 0.100 to 0.999. $\varepsilon_{2}=0.5, \rho_{2}=0.5, I_{b 2}(T)=1$ and $I_{b}(T)=1$. We consider a mesh with $M=30$ and $N=100$. Tables 6 , 7 and 8 show the obtained numerical values of $q^{+}(b)$ for forward, isotropic and backward scattering compared with the ones in the Table 4 of Abulwafa and Attia (1997). In the result tables, REF and P.M. refer to "Abulwafa and Attia (1997)" and "Present Methodology", respectively.
Table 3 - Results for $q^{+}(b)$ and $-q^{-}(a)$, case 1 , with forward scattering ( $\beta_{\ell}$ from Table 1 ) and albedos given by the index $j$ from Table 2 .

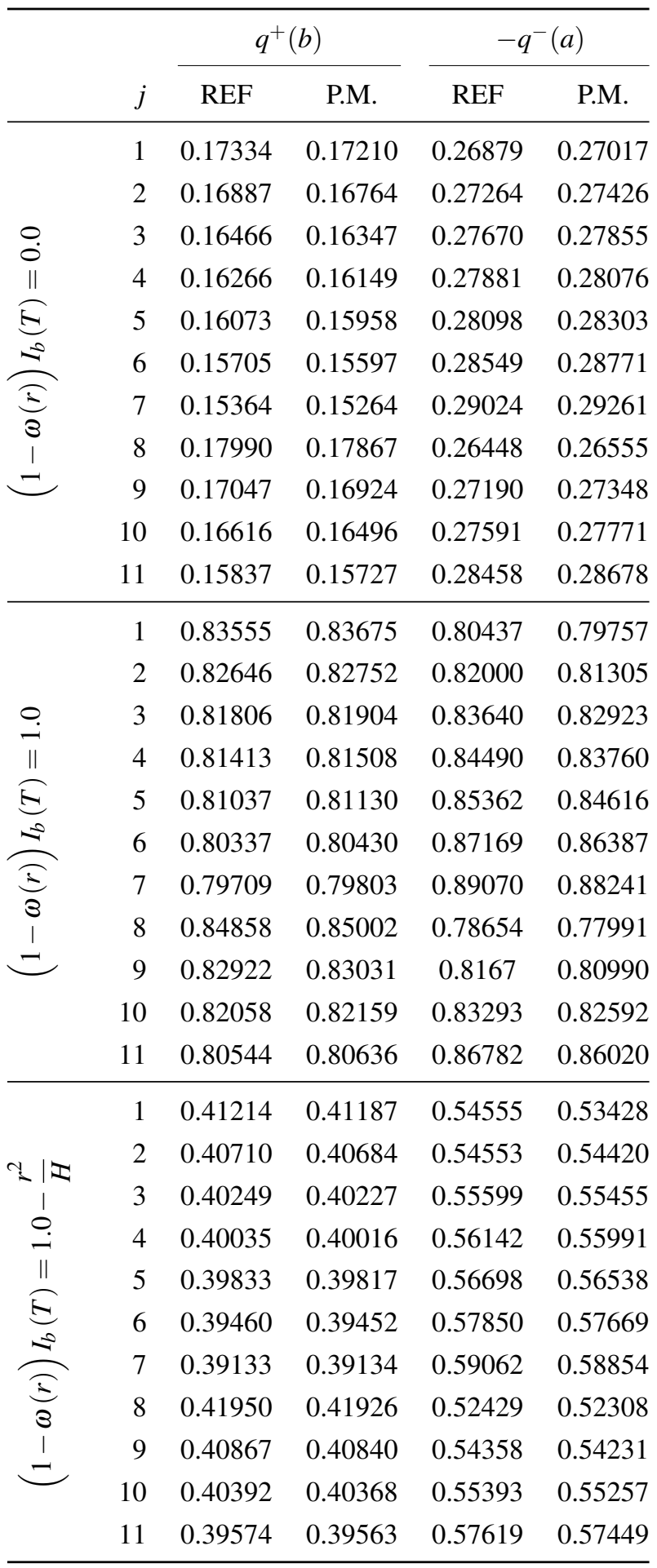

Source: The authors. 
Table 4 - Results for $q^{+}(b)$ and $-q^{-}(a)$, case 1 , with isotropic scattering ( $\beta_{\ell}$ from Table 1$)$ and albedos given by the index $j$ from Table 2 .

\begin{tabular}{|c|c|c|c|c|c|}
\hline & \multirow[b]{2}{*}{$j$} & \multicolumn{2}{|c|}{$q^{+}(b)$} & \multicolumn{2}{|c|}{$-q^{-}(a)$} \\
\hline & & REF & P.M. & REF & P.M. \\
\hline \multirow{11}{*}{ 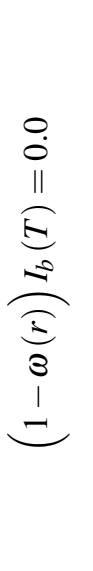 } & 1 & 0.19633 & 0.19692 & 0.23659 & 0.23680 \\
\hline & 2 & 0.19069 & 0.19128 & 0.23028 & 0.23949 \\
\hline & 3 & 0.18531 & 0.18590 & 0.24221 & 0.24243 \\
\hline & 4 & 0.18273 & 0.18331 & 0.24378 & 0.24400 \\
\hline & 5 & 0.18021 & 0.18079 & 0.24541 & 0.24563 \\
\hline & 6 & 0.17537 & 0.17594 & 0.24888 & 0.24911 \\
\hline & 7 & 0.17078 & 0.17135 & 0.25266 & 0.25289 \\
\hline & 8 & 0.20451 & 0.20512 & 0.23373 & 0.23394 \\
\hline & 9 & 0.19275 & 0.19337 & 0.23876 & 0.23898 \\
\hline & 10 & 0.18728 & 0.18787 & 0.24163 & 0.24185 \\
\hline & 11 & 0.17715 & 0.17773 & 0.24817 & 0.24839 \\
\hline \multirow{11}{*}{ 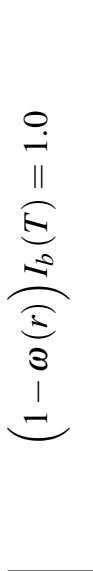 } & 1 & 0.83979 & 0.84053 & 0.78085 & 0.78145 \\
\hline & 2 & 0.83025 & 0.83097 & 0.79472 & 0.79529 \\
\hline & 3 & 0.82139 & 0.82211 & 0.80953 & 0.81010 \\
\hline & 4 & 0.81723 & 0.81794 & 0.81733 & 0.81788 \\
\hline & 5 & 0.81325 & 0.81395 & 0.82540 & 0.82594 \\
\hline & 6 & 0.80579 & 0.80650 & 0.84236 & 0.84289 \\
\hline & 7 & 0.79906 & 0.79975 & 0.86053 & 0.86104 \\
\hline & 8 & 0.85350 & 0.85426 & 0.76512 & 0.76573 \\
\hline & 9 & 0.83322 & 0.83395 & 0.79152 & 0.79208 \\
\hline & 10 & 0.82418 & 0.82485 & 0.80610 & 0.80666 \\
\hline & 11 & 0.80806 & 0.80877 & 0.83840 & 0.83893 \\
\hline \multirow{11}{*}{ 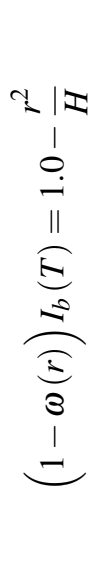 } & 1 & 0.42341 & 0.42402 & 0.51416 & 0.51460 \\
\hline & 2 & 0.41752 & 0.41812 & 0.52325 & 0.52369 \\
\hline & 3 & 0.41206 & 0.41266 & 0.53296 & 0.53340 \\
\hline & 4 & 0.40950 & 0.41010 & 0.53807 & 0.53850 \\
\hline & 5 & 0.40706 & 0.40764 & 0.54335 & 0.54377 \\
\hline & 6 & 0.40249 & 0.40307 & 0.55445 & 0.55487 \\
\hline & 7 & 0.39838 & 0.39895 & 0.56634 & 0.56674 \\
\hline & 8 & 0.43201 & 0.43264 & 0.50393 & 0.50438 \\
\hline & 9 & 0.41948 & 0.42009 & 0.52126 & 0.52169 \\
\hline & 10 & 0.41387 & 0.41448 & 0.53082 & 0.53125 \\
\hline & 11 & 0.40400 & 0.40459 & 0.55198 & 0.55240 \\
\hline
\end{tabular}

Source: The authors.
Table 5 - Results for $q^{+}(b)$ and $-q^{-}(a)$, case 1 , with backward scattering ( $\beta_{\ell}$ from Table 1$)$ and albedos given by the index $j$ from Table 2 .

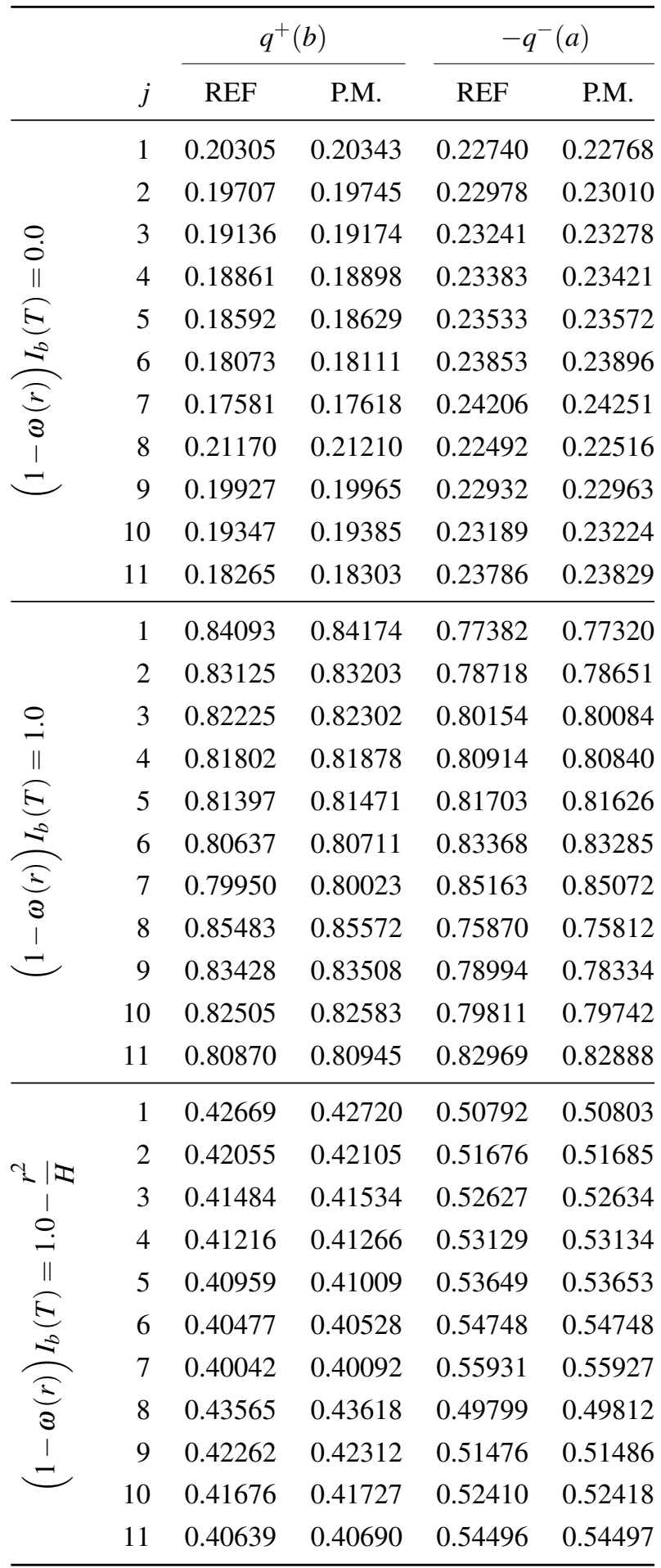

Source: The authors. 
Table 6 - Results for $q^{+}(b)$, case 2, with forward scattering ( $\beta_{\ell}$ from Table 1 ) and several values of $b$.

\begin{tabular}{|c|c|c|c|c|c|c|}
\hline \multirow[b]{2}{*}{$\omega(r)$} & \multicolumn{2}{|c|}{$b=0.1$} & \multicolumn{2}{|c|}{$b=1.0$} & \multicolumn{2}{|c|}{$b=10.0$} \\
\hline & REF & P.M. & REF & P.M. & REF & P.M. \\
\hline 0.100 & 0.511215 & 0.512020 & 0.544716 & 0.544995 & 0.619337 & 0.555623 \\
\hline 0.300 & 0.534881 & 0.535722 & 0.657360 & 0.657857 & 0.755433 & 0.710358 \\
\hline 0.500 & 0.560347 & 0.561229 & 0.818226 & 0.819105 & 0.999109 & 0.978411 \\
\hline 0.700 & 0.587821 & 0.588748 & 1.064922 & 1.066358 & 1.523777 & 1.557299 \\
\hline 0.900 & 0.617543 & 0.618519 & 1.487022 & 1.489217 & 3.574035 & 3.777158 \\
\hline 0.990 & 0.631725 & 0.632725 & 1.792221 & 1.794336 & 10.74023 & 10.88715 \\
\hline 0.999 & 0.633172 & 0.634175 & 1.879144 & 1.831212 & 13.43572 & 13.47837 \\
\hline
\end{tabular}

Source: The authors.

Table 7 - Results for $q^{+}(b)$, case 2, with isotropic scattering ( $\beta_{\ell}$ from Table 1) and several values of $b$.

\begin{tabular}{|c|c|c|c|c|c|c|}
\hline \multirow[b]{2}{*}{$\omega(r)$} & \multicolumn{2}{|c|}{$b=0.1$} & \multicolumn{2}{|c|}{$b=1.0$} & \multicolumn{2}{|c|}{$b=10.0$} \\
\hline & REF & P.M. & REF & P.M. & REF & P.M. \\
\hline 0.100 & 0.511214 & 0.512019 & 0.544598 & 0.544855 & 0.626033 & 0.555202 \\
\hline 0.300 & 0.534874 & 0.535714 & 0.656075 & 0.656436 & 0.768494 & 0.704883 \\
\hline 0.500 & 0.560331 & 0.561212 & 0.814216 & 0.814782 & 0.998583 & 0.954174 \\
\hline 0.700 & 0.587800 & 0.588726 & 1.056900 & 1.057830 & 1.464033 & 1.465998 \\
\hline 0.900 & 0.617531 & 0.618506 & 1.478574 & 1.480097 & 3.102848 & 3.311310 \\
\hline 0.990 & 0.631723 & 0.632723 & 1.790732 & 1.792728 & 9.790239 & 10.01114 \\
\hline 0.999 & 0.633172 & 0.634175 & 1.828986 & 1.831040 & 13.28207 & 13.31584 \\
\hline
\end{tabular}

Source: The authors.

Table 8 - Results for $q^{+}(b)$, case 2, with backward scattering ( $\beta_{\ell}$ from Table 1$)$ and several values of $b$.

\begin{tabular}{|c|c|c|c|c|c|c|}
\hline \multirow[b]{2}{*}{$\omega(r)$} & \multicolumn{2}{|c|}{$b=0.1$} & \multicolumn{2}{|c|}{$b=1.0$} & \multicolumn{2}{|c|}{$b=10.0$} \\
\hline & REF & P.M. & REF & P.M. & REF & P.M. \\
\hline 0.100 & 0.511214 & 0.512018 & 0.544560 & 0.544814 & 0.627975 & 0.555079 \\
\hline 0.300 & 0.534871 & 0.535712 & 0.655673 & 0.656031 & 0.774639 & 0.703408 \\
\hline 0.500 & 0.560326 & 0.561207 & 0.812965 & 0.813555 & 1.002068 & 0.948234 \\
\hline 0.700 & 0.587794 & 0.588719 & 1.054380 & 1.055400 & 1.451819 & 1.445996 \\
\hline 0.900 & 0.617527 & 0.618502 & 1.475805 & 1.477445 & 3.012435 & 3.220619 \\
\hline 0.990 & 0.631723 & 0.632723 & 1.790271 & 1.792254 & 9.520634 & 9.800874 \\
\hline 0.999 & 0.633172 & 0.634175 & 1.828937 & 1.830995 & 13.22896 & 13.27063 \\
\hline
\end{tabular}

Source: The authors.

Case 3 - Refinement

In order to obtain good results with the present methodology we had to use high values for $M$ and $N$, as some $I_{m}^{i}$ were less than zero for small $M$ and $I$, which has no physical meaning. In the Tables 9 and 10 we show the effects of $N$ (spacial) and $M$ (angular) refining for a solid sphere with numerical values $a=0, b=10.0$, a forward scattering $P$ of Table $1, \omega(r)=0.100, \varepsilon_{2}=1.0$, $\rho_{2}=0.0, I_{b 2}(T)=1$ and $I_{b}(T)=0$. 
Table 9 - Some values of $I_{m}^{N}$ for the case 3, $N=100$ and different values of $M$.

\begin{tabular}{lcccc}
\hline$m$ & $M=8$ & $M=18$ & $M=30$ & $M=36$ \\
\hline 1 & 1.000000 & 1.000000 & 1.000000 & 1.000000 \\
2 & 1.000000 & 1.000000 & 1.000000 & 1.000000 \\
3 & 1.000000 & 1.000000 & 1.000000 & 1.000000 \\
$\vdots$ & $\vdots$ & $\vdots$ & $\vdots$ & $\vdots$ \\
$M-2$ & -0.036613 & 0.005473 & 0.001724 & 0.001056 \\
$M-1$ & 0.026204 & -0.000430 & 0.000832 & 0.001089 \\
$M$ & -0.008700 & 0.001907 & 0.000999 & 0.000817 \\
\hline
\end{tabular}

Source: The authors.

Table 10 - Some values of $I_{m}^{0}$ for the case 3, $M=30$ and different values of $N$.

\begin{tabular}{lrccc}
\hline$m$ & $N=10$ & $N=20$ & $N=50$ & $N=100$ \\
\hline 1 & -0.000716 & 0.000018 & 0.000082 & 0.000084 \\
2 & -0.001304 & -0.000021 & 0.000081 & 0.000084 \\
3 & -0.001395 & -0.000028 & 0.000081 & 0.000084 \\
$\vdots$ & $\vdots$ & $\vdots$ & $\vdots$ & $\vdots$ \\
$M-2$ & -0.001395 & -0.000028 & 0.000081 & 0.000084 \\
$M-1$ & -0.001304 & -0.000021 & 0.000081 & 0.000084 \\
$M$ & -0.000716 & 0.000018 & 0.000082 & 0.000084 \\
\hline
\end{tabular}

Source: The authors.

\section{Concluding remarks and future work}

This article is about a simple methodology that is able to solve many cases of introdutory radiation transfer, for solid and hollow spheres, inhomogeneous medium and anisotropic scattering with classical boundary conditions. In other words, it is about a methodology that is ready to be expanded to more difficult cases, in the sense that it does not require geometry restriction (solid or hollow sphere) or scattering restriction (isotropic or linearly anisotropic) in any way. It works smooth with and without black body radiation, in the domain and boundaries, and to run the code for most cases, it took about 30 seconds or less in a domestic computer.

We run the code for some classical references (ABULWAFA, 1993; ABULWAFA; ATTIA, 1997). Our numerical values coincided with the references for all cases with the same good agreements as in Tables 3, 4, 5, 6, 7 and 8. We choose to include in this paper the cases we considered to be the most challenging to a numerical iterative scheme.
In cases 1 and 2 we can see that the forward, isotropic and backward scattering types did not have major impact on the partial heat fluxes. The present methodology is not restricted to phase functions like (2), it actually might be used for any kind of $P\left(\mu, \mu^{\prime}\right)$. Also, from case 2 we can see that greater albedos and radius made the greater changes in the numerical values, and from case 1 we note how greater values of the black body radiation impact on the results. The latter requires special attention, as for more realistic cases, the black body radiation is modelled with a nonlinearity in $I(r, \mu)$. In the tables of case 3 , we show where there were the majority of inconsistencies (negative intensities), but there were cases where negative values of $I$ appeared in the middle of the $I_{m}^{i}$ matrix. A curious fact about these incorrect results before the refinement: the values of their partial heat fluxes were, apparently, correct, despite they have been computed with non realistic intensities. This highlights the fact that the intensities must be taken into account for refining purposes, rather than the heat fluxes. Also, as we consider a radiative transfer problem, this method may be easily adapted to other neutral particles transport, like neutrons in a nuclear reactor core.

For future work, we intend to develop a convergence and stability analysis, which is not usual for transport theory papers, even with numerical methods application. Also, the expansion to higher dimensions would imply bigger matrices, which could cause a memory stack overflow. We would like to investigate how to overcome this problem. We also intend to approach some more realistic cases for radiative transfer, which involve coupling this transport equation with a heat conduction equation, making a nonlinear system.

\section{Acknowledgments}

The authors wish to thank FAPERGS - Brazil, for financial support.

\section{References}

ABULWAFA, E. M. Radiative-transfer in a linearlyanisotropic spherical medium. Journal of Quantitative Spectroscopy and Radiative Transfer, [S.l.], v. 49, n. 2, p. 165-175, 1993. DOI: https://doi.org//10.1016/00224073(93)90057-O. 
ABULWAFA, E. M.; ATTIA, M. T. Radiative transfer in a spherical medium by the variational PomraningEddington technique. Journal of Quantitative Spectroscopy and Radiative Transfer, [S.l.] v. 58, n. 1, p. 101-114, 1997. DOI: https://doi.org/10.1016/S00224073(97)00021-6.

CHANDRASEKHAR, S. The Radiative Transfer. New York: Oxford University Press, 1950.

ELGHAZALY, A. Radiative Transfer Equation for Anisotropic Spherical Medium with Specular Reflective Index. In: CONFERENCE ON NUCLEAR AND PARTICLE PHYSICS, 7., 2009, Egyty. Proceeding [...]. Egypt: [s. n.], 2009. p. 291-300. Available from: https://inis.iaea.org/search. Acess on: 31 Mar. 2020.

HOWELL, J. R.; MENGUC, M. P.; SIEGEL, R. Thermal Radiation Heat Transfer. New York: CRC Press, 2016.

LEWIS, E. E.; MILLER JUNIOR, W. F. Computational Methods of Neutron Transport. New York: John Wiley \& Sons, 1984.

MODEST, M. F. Radiative Heat Transfer. New York: Academic Press, 2013.
OZISIK, M. N. Radiative transfer and interaction with conduction and convection. New York: Wiley \& Sons Inc., 1973.

PETTY, G. W. A first course in atmospheric radiation. Madison: Sundog Publishing, 2006.

THYNELL, S.; OZISIK, M. N. Radiation transfer in na isotropically scattering solid sphere with space dependente albedo, w(r). Journal of Heat Transfer, New York, v. 107, n. 3, p. 732-734, 1985. DOI: https://doi.org/10.1115/1.3247491.

TSAI, J. R.; OSIZIK M. N.; SANTARELLI, F. Radiation in spherical symmetry with anisotropic scattering and variable properties. Journal of Quantitative Spectroscopy and Radiative Transfer, [S. l.], v. 42, n. 3, p. 187-199, 1989. DOI: https://doi.org/10.1016/0022-4073(89)900824.

SGHAIER, T. Study of Radiation in Spherical Media Using Moment Method. American Journal of Physics and Applications, New York, v. 1, n. 1, p. 25-32, 2013. DOI: https://doi: 10.11648/j.ajpa.20130101.15. 